\title{
Scientific Principles behind 2017 World Universiade Torch
}

\author{
Wei-Long Chen; Fang-Lin Chao; Syuan-Lan Shih \\ Department of Industrial Design, Chaoyang University of Technology, \\ Wufang Taichung, 436, \\ Taiwan, R.O.C. \\ flin@cyut.edu.tw
}

\begin{abstract}
The innovative torch was revised and tested, follow-up production successfully. The Safety lantern uses biofuel and displays a beautiful spiral flash. Major scientific principles are Stack effect, Coanda effect, and the Capillary phenomenon. There are two types of people involved in the design, and one is the industrial designer: responsible for the presentation of the beautiful material. The other is mechanical engineers. When a problem is solved, other design parameters may be affected; it is necessary to return to the original point to propose a solution to the common solution. The case study presents the importance of cross-discipline collaboration.
\end{abstract}

Key words: scientific principles, torch, convection

\section{Introduction}

\section{A. The event}

The World University Games, known as the Little Olympics, is hosted by the International University Sports Federation and is an international comprehensive sports event for university athletes. It is held in different cities every two years. Taiwan won the right to host the 29th Summer Universiade. This is the highest level and largest international competition held in China; the competition will be held from August 19 to 30, 106. It includes 22 competition types including track and field, tennis, badminton, weightlifting, martial arts, and roller skating. It includes 134 national teams from home and abroad and more than 10,000 players [1]. Competitive sport in colleges and universities either belongs to the part of physical education or belongs to the components of the national competitive sport, which plays two roles on social sport [2].

The transmission of the flame symbolizes the spirit of the athletes' success, which is a process that is more widely watched by the public outside the stadium. Although the conference was held in August, the transmission of the Flame was started in June. The torch relay started on June 20 in the Italian city of Turin [1].

\section{A. Design team}

In order to demonstrate our emphasis on this event, the conference decided to redesign the torch and the mother flame element with reference to Taiwan's culture and Olympic spirit. The design team included Taiwan's essential design elements and craft skills in the design. The concept was revised and tested, follow-up production successfully [2] (Figure 1).

Flames are dangerous and extremely safe objects. The design must meet the functional and safety requirements of the product. Therefore, designers also need to understand the scientific principles in order to work in the design and find the appropriate solution. Industrial designers must interact with mechanical designers and fuel experts to be able to design smoothly.

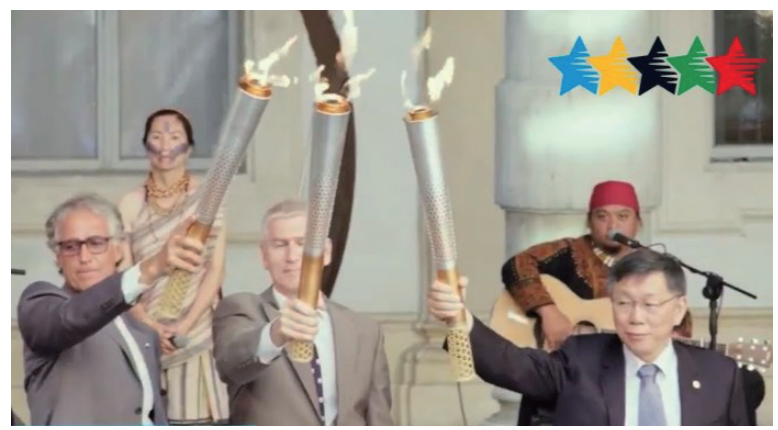

Fig. 1 Torch used in 2017 Taipei Summer Universiade [2]

Figure 2 shows the design concept comes from Confucian's article "Fair play." The torch made of metal, on which there are an array of ventilating holes. The lower holding portion made of weaving bamboo. In the past, bamboo craftsmanship was a traditional family craft, but it has gradually faded away. The ingenious combination of bamboo weaving and high-tech laser cutting metal tubes is a beautiful match. The bamboo braided device has a gap between it and the metal tube, and it has a weak heat conductor, so it is also a suitable heat insulating material. Traditional craftsmanship combines functionality and beauty.

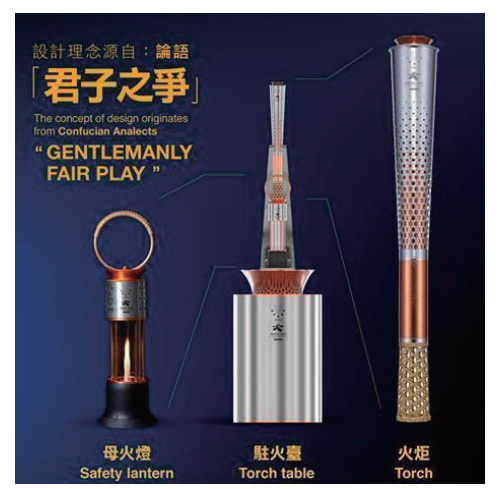

Fig. 2 The concept of "Fair play" was implemented in safety lantern and torch [2].

Scientific principles 
The spiral frame of safety lantern is visible and has a stable shape and an elongated height (Figure 2). The torch produces a swirling flame, and since gas and air mixture undergoes the first and second mixing processes and air flows out of the plurality of passages (L1) spirally, and a stable fluid field created in the guided shield channels. After gas and air mixing process and is being ignited by the igniting needle, combustion flame flowing out from the grooves and fresh air drawn from the passages mix. Heated air in the shield creates buoyancy due to a difference in air density. The atmosphere in the shield driven by buoyancy goes upward and draws in fresh air through the passages which make air flowing therein rotate and go upward.

\section{A. Stack effect}

The Stack effect [3] comes from natural convection in which the fluid motion is not generated by the external source but only by density differences in the fluid occurring due to temperature gradients. We try to keep the flow to be laminar flow in this design by specific guiding channel. The phenomenon and detail structures related to Stack effect shown in Figure 3.

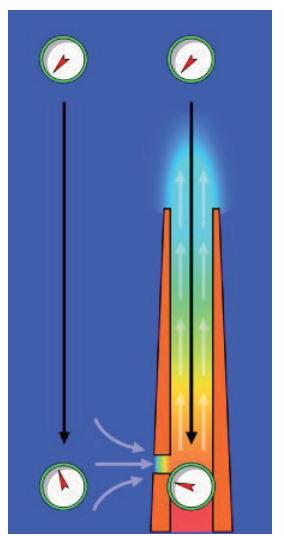

Fig. 3 Stack effect [3] (gauges represent air pressure, airflow indicated with arrows)

Also known as the chimney effect, when inside the towering chimney, the gas heats up and flows upwards. The vacuum formed below moves the inflow of gas from the outside, so it continues to float upwards. We can see the towering round tubular glass column in the design of the mother fire. The purpose is to create a chimney effect, allowing the heated gas to flow upwards, allowing the outside cold air to enter from the lower guide piece. This steady flow creates a higher flame that increases the aesthetics of the viewing.

\section{B. Coanda effect and centrifugal force effect}

The passages direct fresh air toward the inner surface of a shield to create a spiral air flow due to Coanda effect and centrifugal force effect. Coanda effect [4] related to the tendency of a fluid jet to stay attached to a convex surface.

The guiding airflow slowly flows upward along the guiding piece, and this rotating airflow tends to adhere to the inside of the glass tube and continues to rotate upward. Although the movement of this airflow is not apparent, the cold air thus applied can effectively carry away the heat energy at the glass interface. The temperature of the surface of the glass cylinder is lowered, so it is a significant niche regarding safety.

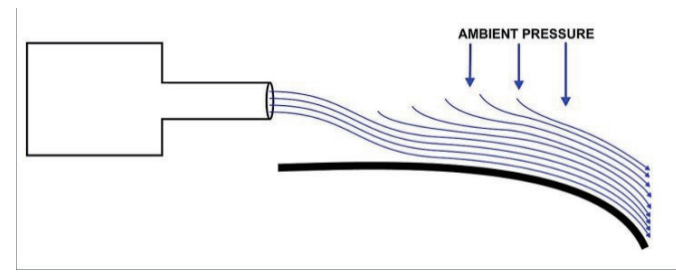

Fig. 4 The jet flow adheres to the nearby surface even a curved surface [4].

The fluid within torch is the mixture of gas and air. The fluid stream attaches a contour when the flow is directed at a tangent to that surface. The centrifugal force is an inertial force led away from the axis of rotation that appears to act on objects when viewed in a rotating reference axis $[5,6]$. Based on the formula of centrifugal force, the particles far away from the center have a more significant speed. The detail of the structure in the torch shows in Figure 5.

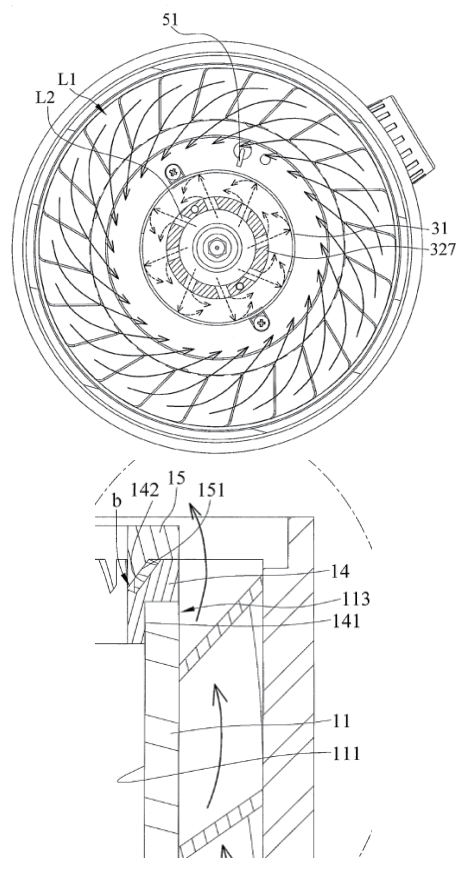

Fig. 5 Flow of air in the flow diverting mechanism, note that the gas outlet in the central cylinder is not perpendicular to the surface.

\section{Capillary phenomenon}

The capillary phenomenon is the ability of a liquid to flow in narrow spaces in opposition to gravity $[7,8]$. The fundamental equation of capillarity specifies the configuration of these fluid-fluid interfaces. Fluid-fluid interfaces are found within a certain fraction of the pore openings. The thinner the space in 


\section{Recent Developments on Information and Communication Technology (ICT) Engineering-Meen, Yang \& Zhao ISBN: 978-981-14-2136-5}

which the liquid can travel, the further up it goes.

The cord placed in the holding space and the two jaws can hold the wick so that the wick's head end located in the hot area $[9,10]$. By adjusting the gap when clamping the cord will compress and modify the fuel feeding and the size of the flame [11].

The fuel used in the mother fire is a biomass fuel. Because of its high ignition point, complete combustion achieved with proper mixing. In the process of the torch relay, we need to keep the mother fire in a burning state, which means the continuous transmission. A stable and continuous source of fire is also required. Aircraft required to pass the flames across the continent today. We know that the plane is a confined space and there is a risk of poisoning if carbon monoxide produced during the combustion process. Here we can avoid such problems by using a stainless steel grid fuel delivery.

\section{Evaluation}

\section{A. Mother flame}

Figure 6 indicates the assessment of the experimental prototype. We found several facts. The flame guiding member allows controlling convection between the secondary gas mixing and fresh air drawn from outside the chamber. After gas and air mixture has thoroughly mixed in the mixing chamber and the pressure difference is stabled, a gas and air mixture flows exist between the outside of the chamber and the guiding grooves. The angle of skew was guiding members inference the flame significantly (Figure 6).

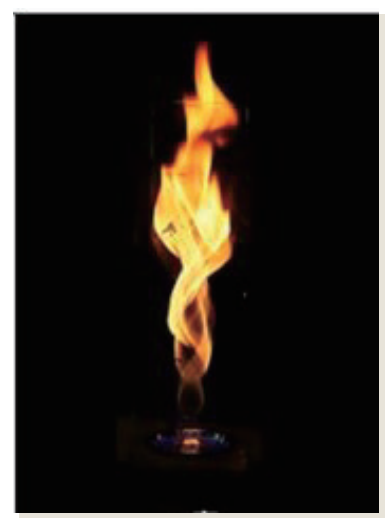

Fig. 6 Evaluation of an experimental prototype

B. Torch

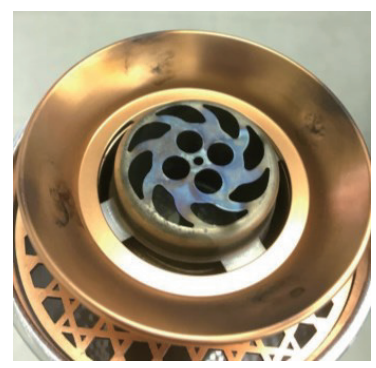

(a)

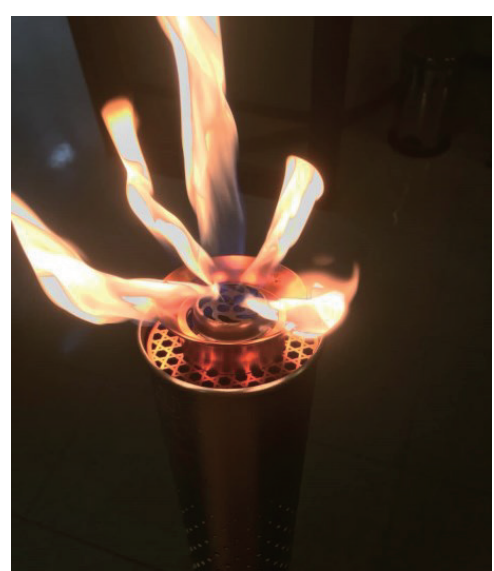

(b)

Fig. 7 The flow diverting mechanism design of the torch

Figure 7 and 1 show pattern of five flames. The main body of the fire moment is a hollow metal rod made of aluminum, and below it is a mixing line of the gas storage tank and intake air flow. The high-pressure gas is sprayed upward from the metal line. In the upper half of the mixing chamber combined with air, a flame is ejected from the top metal cover. The round hole in Figure 7(a) is the main flame exit; the flame outlet at the side also forms a large four small five flames, showing strong energy and light heat. When the outside air enters the air mixing chamber since the outside air is 30 degrees or less an average temperature, the intake air adsorbed therein can also be moved during the flow through the metal pipe. Cooling the aluminum rod also eliminates the chance of burns when it comes into contact with the outside of the torch.

\section{C. heat transfer phenomenon}

The heat transfer divided in three ways: heat conduction, heat convection, and heat radiation. Among them, when the temperature difference is significant, heat radiation is the primary mode of transmission. Both heat conduction and heat radiation transfer heat energy in all directions; conversely, heat convection usually transports heat energy upward. When heat radiation is applied, it is not necessary to transmit any medium matter, just like electromagnetic waves, the heat energy is transferred.

We saw that the glamorous metal engraving shell was made using laser engraving. Laser engraving can engrave correct paths. However, this is only the outermost metal. The aluminum tube is the main combustion component area in the inner layer. There is a slight air gap between the inner and outer metal tubes. Because the thermal conductivity of steady air is low, internal heat cannot be transferred smoothly to the outer casing.

Emissivity is the ability of a surface of a material to penetrate (absorb) radiant energy. The penetration force (E value) ranges from 0 to 1 , and the closer the $\mathrm{E}$ value is to 0 , the worse the penetration rate is. An object with an emissivity of 0.8 can absorb $80 \%$ of the incoming energy while reflecting the other $20 \%$.

Between the inner and outer two metal tubes, it is possible to transmit via radiation because of the high-temperature 
difference. We need to consider the surface treatment of the metal. When the surface of the metal is not rough or dark, the pattern of bright reflection can significantly reduce the rate of absorption of heat radiation.

\section{Conclusions}

The case study discussed mainly the scientific principles behind the design. The spiral frame of safety lantern exhibits a stable shape and an elongated height. Three scientific principles are Stack effect, Canada effect, and Capillary phenomenon.

In the process of design, we found that there are two types of people involved in the design, one is the industrial designer: responsible for the presentation of the aesthetic material. The other type is mechanical engineers: accountable for the achievement of functions, the organization of the organization and the rationality of manufacturing. The two teams are dependent on each other. For example, we need to burn completely to reduce the carbon monoxide content and avoid poisoning. Secondly, in the case of high heat, we need to use heat conduction to walk the tropics to prevent burns. So a single problem requires teamwork.

In the idealized case, the function is completed first, followed by the styling design. However, the real situation and non-linear execution are often cyclic. When a problem is solved, other design parameters may be affected; it is necessary to return to the original point to propose a solution to the standard solution. This is an essential of multi-domain interactive design.

\section{References}

[1] https://www.bbc.com/zhongwen/trad/chinese-news-41105319

[2] Jiang, Ling, Zhong-qing Liu, and Jian-hua Chen. "Research on Construction and Development of Competitive Sports Administration System in Colleges and Universities," Journal of Beijing University of Physical Education, Vol. 4, 2001.

[3] https://en.wikipedia.org/wiki/Stack_effect

[4] https://en.wikipedia.org/wiki/Coand $\%$ C4\%83_effect

[5] Marques, Francisco, Isabel Mercader, Oriol Batiste, and Juan M. Lopez. "Centrifugal effects in rotating convection: axisymmetric states and three-dimensional instabilities." Journal of Fluid Mechanics 580 (2007): 303-318.

[6] Enger, Sven, Biswajit Basu, Michael Breuer, and Franz Durst. "Numerical study of three-dimensional mixed convection due to buoyancy and centrifugal force in an oxide melt for Czochralski growth," Journal of Crystal Growth, Vol. 219, no. 1-2, 2000, pp.144-164.

[7] Morrow, Norman R. "Physics and thermodynamics of capillary action in porous media." Industrial \& Engineering Chemistry 62.6 (1970): 32-56.

[8] Hocking, L. M., and A. D. Rivers. "The spreading of a drop by capillary action," Journal of Fluid Mechanics, Vol. 121, 1982 , pp.425-442.

[9] US Patent: US20120178035, Device for producing stable and augmented flame.

[10] US Patent: US20130252188, Flame device.

[11] Taiwan Patent: I529335, Modular core fixing device and its support unit. 